\title{
THE DESIGN OF DATA-STRUCTURE-NEUTRAL LIBRARIES FOR THE ITERATIVE SOLUTION OF SPARSE LINEAR SYSTEMS
}

\author{
BARRY F. SMITH * WILLIAM D. GROPP ${ }^{\dagger}$
}

March 9, 1993

\begin{abstract}
Over the past few years several proposals have been made for the standardization of sparse matrix storage formats in order to allow for the development of portable matrix libraries for the iterative solntion of linear systems. We feel that this is the wrong approach. Rather than define one standard (or a small number of standards) for matrix storage, the community should define an interface (i.e., the calling sequences) for the functions that act on the data. In addition, we cannot ignore the interface to the vector operations since, in many applications, vectors may not be stored as consecutive elements in memory. With the acceptance of shared-memory, distributed-memory, and cluster-memory parallel machines, the flexibility of the distribution of the elements of vectors is also extremely important.
\end{abstract}

Key words. Krylov space methods, software libraries, sparse linear systems

AMS(MOS) subject classifications. $65 \mathrm{~F} 10,65 \mathrm{~F} 50,68 \mathrm{~N} 05$

\section{Introduction}

In the 1970s two extremely successful numerical linear algebra software packages, EISPACK and LINPACK, were introduced. They were designed for portability, generality, numerical robustness, and efficiency. They were, however, restricted to dense and banded matrices. The development of serial numerical linear algebra software for dense and banded matrices is greatly simplified by the fact that there are very few natural ways of storing the matrices. Thus very little effort is needed in designing the data structures used in the codes.

- Department of Mathematics, University of California, Los Angeles, CA 90025-1555. Electronic mail address: bsmith(ồmath.ucla.edu. This work was supported in part by the Applied Mathematical Sciences subprogram of the Office of Energy Research, U.S. Department of Energy, under Contract W-31-109-Eng-38 while the author was at the Argonne National Laboratory, and by the Office of Naval Research under coutract ONR No0014-90-J-1695.

'Argonne National Laboratory, 9700 South Cass Ave., Argonne, Il 60439-4844. Electronic mail address: gropp Department of Energy, under Contract W-31-109-Eng-38. 


\section{DISCLAIMER}

This report was prepared as an account of work sponsored by an agency of the United States Government. Neither the United States Government nor any agency thereof, nor any of their employees, make any warranty, express or implied, or assumes any legal liability or responsibility for the accuracy, completeness, or usefulness of any information, apparatus, product, or process disclosed, or represents that its use would not infringe privately owned rights. Reference herein to any specific commercial product, process, or service by trade name, trademark, manufacturer, or otherwise does not necessarily constitute or imply its endorsement, recommendation, or favoring by the United States Government or any agency thereof. The views and opinions of authors expressed herein do not necessarily state or reflect those of the United States Government or any agency thereof. 


\section{DISCLAIMER}

Portions of this document may be illegible in electronic image products. Images are produced from the best available original document. 
For sparse linear algebra, even on sequential machines, the issues become much more complicated. When one includes various parallel machines, the problems multiply even further. Not only must one make decisions about the storage of the sparse matrices, one must also decide on storage formats for the vectors, since each vector is probably distributed across the parallel processors. We also note that even on sequential machines, the natural storage format for a vector should be dictated by the application. For instance, an adaptive mesh refinement code may represent the solution and other vectors with an octtree data structure.

Software methodologies to overcome these problems do exist; they involve data hiding and object-oriented programming techniques. In object-oriented programming one abstracts out of a data type the actions that one wishes to perform on the data, independent of the underlying representation of the data. So, for instance, in the iterative solution of linear systems one needs to be able to multiply vectors by sparse matrices and their transposes. In addition, one must be able to perform scalings of vectors, calculate sums of vectors, etc. These operations, not the particular representation of the matrices and vectors, are what define the data. Thus any storage format, with the corresponding operations defined, should be immediately supported by the software library.

To someone used to programming in Fortran 77, this may sound like a pipe dream. It is actually relatively easily achieved in some programming languages. In this paper we describe an implementation using $C$, since many people are familiar with this language and it is portable and available on virtually all machines. Also, it is fairly easy to mix Fortran 77 and $\mathrm{C}$ code in a single application on most platforms.

Note that some people use the term object-oriented to refer to specifying a data type, operations on that data type, and all of the details of the internal formats (for example, the sparse matrix format to use). We are using object-oriented in a stronger and purer sense: only the operations are specified. The choice of internal format (and hence, the choice of the actual code to implement the operations) is determined only at run time rather than at compile time. This is an important difference; it changes object-oriented from being simply a way to organize a code and the argument lists of the routines to a method for flexibly adapting to different situations.

\section{Programmer Defined Data Types}

In Fortran 77 a limited number of data types are built into the language, essentially scalar integer and floating-point numbers, and dense arrays of integer and floatingpoint numbers. The language contains no mechanism for the programmer to construct additional data types. Hence, when dealing with higher-level objects such as sparse matrices, the programmer must choose a particular storage format, which, in general, will involve several separate array variables. All of these array variables must be passed to the routines that operate on the sparse matrices.

To explain this more fully, we give a particular example, the well-known Yale Sparse Matrix Package (YSMP) storage scheme [2]. In YSMP the sparse matrix is stored by using four variables: $n$, the size of the matrix; $a$, an array of floating-point numbers that contain the nonzero entries in the matrix; $i a$, an array of integers that contain the locations in a of the beginning of each new row; and $j a$, which contains the column number of each entry in a. A variation of this storage format is to store the diagonal entries separately in another array, $d$.

If one desired to write a general-purpose iterative solver routine that used the 
YSMP storage pattern, it could have a calling sequence like $C G(n, a, i a, j a, \ldots)$. But if one desired to support both storage formats, one would need something like $\operatorname{CG}(n, a, i a, j a, d, f l a g, \ldots)$, where the value of flag indicates which of the two formats is being used. This increases the complexity of the code and makes the addition of a new storage format difficult: it may require not only rewriting the CG() code, but also modifying all of the application codes, since the calling sequence of the CG() code has been changed.

Other programming languages such as $\mathrm{C}, \mathrm{C}++$, and Fortran 90 provide a better and more flexible alternative. The programmer is free to introduce new data types, called structures in $\mathrm{C}$, classes in $\mathrm{C}++$, and derived data types in Fortran 90. One feature that is useful about these new data types is that pointers to the data may be passed into a routine without the routine needing to know what information they contain and how it is stored. In this way the CG() routine need not know the storage format of the matrix; only the matrix-multiply routine needs to know it. So, for instance, one may introduce a new data type, Sparsellatrix, then write conjugate gradient routines like the following that will support any matrix storage format.

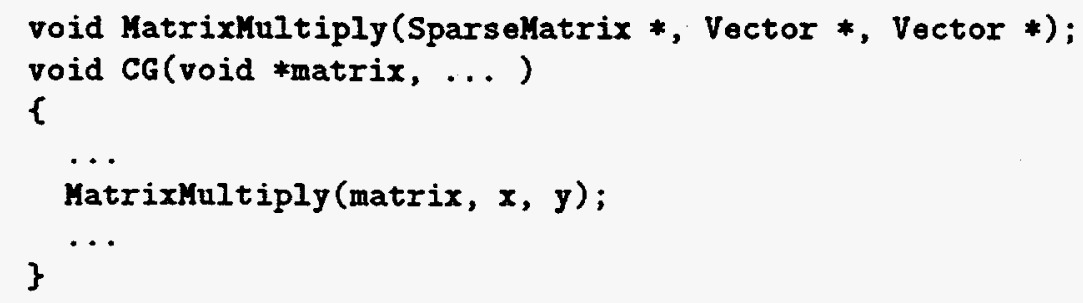

For those not familiar with C's void type, this simply means that the data type is unspecified. If the sparse matrix storage format is changed, only the MatrixMultiply() routine must be changed, not the $\mathrm{CG}()$ routine. In fact, we can do even better than this. Rather than hardwiring into the $C G()$ code the matrix-multiply routine, we can pass a pointer to the matrix multiply routine into the $\mathrm{CG}()$ routine.

\section{Our Approach}

Since we would like to support a variety of Krylov-based solvers, we must first determine which vector operations these require. Some of these are the standard BLAS 1 operations. Others include routines to generate and free vectors that are needed for temporary or permanent workspace. Since it would be cumbersome to individually pass pointers to all of these routines into the solver routines, we bundle up all of the function pointers and any additional data needed for a particular implementation into a single data type, called a vector context, VectorContext. In Fig. 1 we give a part of our (; structure that defines the VectorContext. All higher-level routines that require access to the vectors act on the vectors only through the vector context, not by directly manipulating the data.

All of the vector routines take, as their first argument, a pointer to a private, implementation-dependent data structure that may contain the vector length, layout, etc. For a standard serial vector implementation this can simply be a pointer to an integer containing the length of the vector. For a simple parallel implementation it may be a pointer to two integers, the first containing the length of the part of the vector stored in local memory, the second the length of the entire vector. A sample 


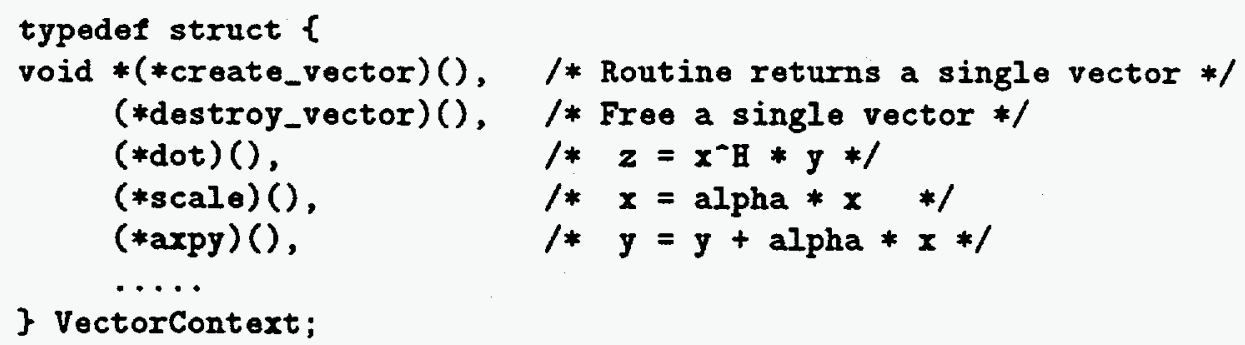

Figure 1: The Vector Context

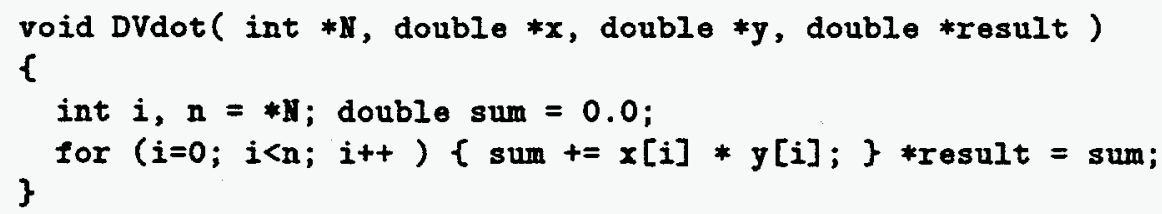

Figure 2: A Sample Dot Product

serial implementation of the $\operatorname{dot}()$ routine is given in Fig. 2. Associated with each vector operation is a macro to simplify its use; for instance, to use the vector $\operatorname{dot}()$ operation one may use VDOT (vp, $y, x, y$, result).

Currently, our vector structure provides the operations from the Level 1 BLAS, plus the operations $y \leftarrow x+\alpha y$ and $w \leftarrow \alpha x+y$, along with operations to create and free storage for vectors. In Table 1 we list the minimal vector operations we feel must be defined. Note that the first argument is defined to be a pointer to void. The pointers to Scalar or Vector are also unspecified; the indication Scalar or Vector is there simply to allow type checking of arguments for those languages that support it. These calling sequences will allow the same codes to be used with single precision, double precision, complex, multiple precision, interval arithmetic, etc.

Sparse matrix operations may be stored similarly; in addition to the obvious operations such as matrix-vector product and triangular solve, we include such operations as insert and extract row and compute incomplete factorizations. Sparse matrices have a similar table, which, to keep this article short, will not be displayed here.

An important feature of the data-hiding approach is that additional operations can be added without disturbing existing code. For example, the operation $w \leftarrow \alpha x+y$ was added when it became apparent that several Krylov methods could make good use of it. The previously coded Krylov space methods did not require any changes. If these routines were passed through argument lists (the only portable mechanism available for Fortran 77 programmers), adding a routine would require modifying each argument list for every routine that used these vector routines.

The only technique available to Fortran programmers that approximates this flexibility is "reverse communication." In this method, for each operation, the library routine sets a flag and returns to the calling program with a request that an operation be performed. However, this method puts the burden on the user, as well as requiring a rather unnatural style of programming. 
Table 1: Vector Operations

\begin{tabular}{|c|c|c|}
\hline Name & Description & Calling Sequence (first argument is always void $* \mathrm{~N}$ ) \\
\hline Create & a vector & \\
\hline Destroy & a vector & Vector $* v$ \\
\hline Obtain & n vectors & int $n$ \\
\hline Release & n vectors & int $\mathrm{n}_{1} \quad$ Vector ${ }^{* *} \mathrm{v}$ \\
\hline Dot & $z \leftarrow x^{H} * y$ & Vector $*_{\mathrm{x}}$, Vector $*_{\mathrm{y}}$, Scalar $*_{\mathrm{z}}$ \\
\hline Norm & $z \leftarrow \sqrt{x^{H} * x}$ & Vector $*_{\mathrm{x}}$, Scalar $*_{\mathrm{z}}$ \\
\hline Max & $z \leftarrow \max (|x|)$ & Vector $*_{\mathrm{x}}$, Scalar $*_{\mathrm{z}, \text { int }} *_{\mathrm{idx}}$ \\
\hline Scale & $x \leftarrow \alpha x$ & Scalar ${ }^{*} \alpha$, Vector ${ }^{*} \mathrm{x}$ \\
\hline Copy & $y-x$ & Vector ${ }^{*} \mathrm{x}$, Vector ${ }^{*} \mathrm{y}$ \\
\hline Set & $x_{i} \leftarrow \alpha, \forall i$ & Scalar $* \alpha$, Vector $* x$ \\
\hline AXPY & $y \leftarrow \alpha x+y$ & Scalar $*_{\alpha}$, Vector ${ }^{*} \mathrm{x}$, Vector ${ }^{*} \mathrm{y}$ \\
\hline AYPX & $y \leftarrow \alpha y+x$ & Scalar $* \alpha$, Vector ${ }^{*} \mathrm{x}$, Vector ${ }^{*} \mathrm{y}$ \\
\hline Swap & swap $x$ and $y$ & Vector $*_{x}$, Vector $*_{y}$ \\
\hline WAXPY & $w \leftarrow \alpha x+y$ & Scalar ${ }^{*} \alpha$, Vector ${ }^{*} \mathrm{x}$, Vector ${ }^{*} \mathrm{y}$, Vector ${ }^{*} \mathrm{w}$ \\
\hline
\end{tabular}

Since the various Krylov-based solvers have many optional arguments, we use a context data type, IterativeContext, to store this information as well as the location of the right-hand side, solution, etc. The IterativeContext has two parts, a public part which is the same for all Krylov space methods, and a private part which contains particular options, workspace, etc., for each particular Krylov space method. The distinction between the two parts is invisible to the application programmer. The user may also provide optional routines to replace the default convergence tests and optional routines to print out or plot the solution, residual, error, etc., at each iteration; these are stored in the IterativeContext, as well. The IterativeContext also contains a VectorContext for use with operations on the vectors.

In Fig. 3 we show an implementation of the inner loop of a preconditioned conjugate gradient. This implementation is portable and works correctly on parallel computers regardless of the distribution of data (all of the difficulty is handled by the specific choices of functions for the vector and matrix operations). In fact, it is taken from the version that we are currently using on both uniprocessors and parallel computers such as the Intel DELTA and BBN TC2000.

In Fig. 4 we give a code fragment that will allow the solution of a linear system using the conjugate gradient method, GMRES, Bi-CG-stab, CGS, or two different versions of transpose-free QMR. The first line currently configures the code for the GMRES method. The important point is that all of the different methods have the same calling sequences. Optional arguments are passed by calling additional routines, which are ignored if the option is not appropriate. In this way any of the methods in the library may be used without changing the application code at all. In addition, more Krylov space methods may be added to the library without a need for any changes to the application codes. Of course, this flexibility is purchased at a price. Adding a method requires following the object-oriented approach. Further, the routines amult () and binv() must be provided by the user, and they must also conform to the implementation, though normally a library would provide several default implementations. It has been our experience that the object-oriented design makes this 


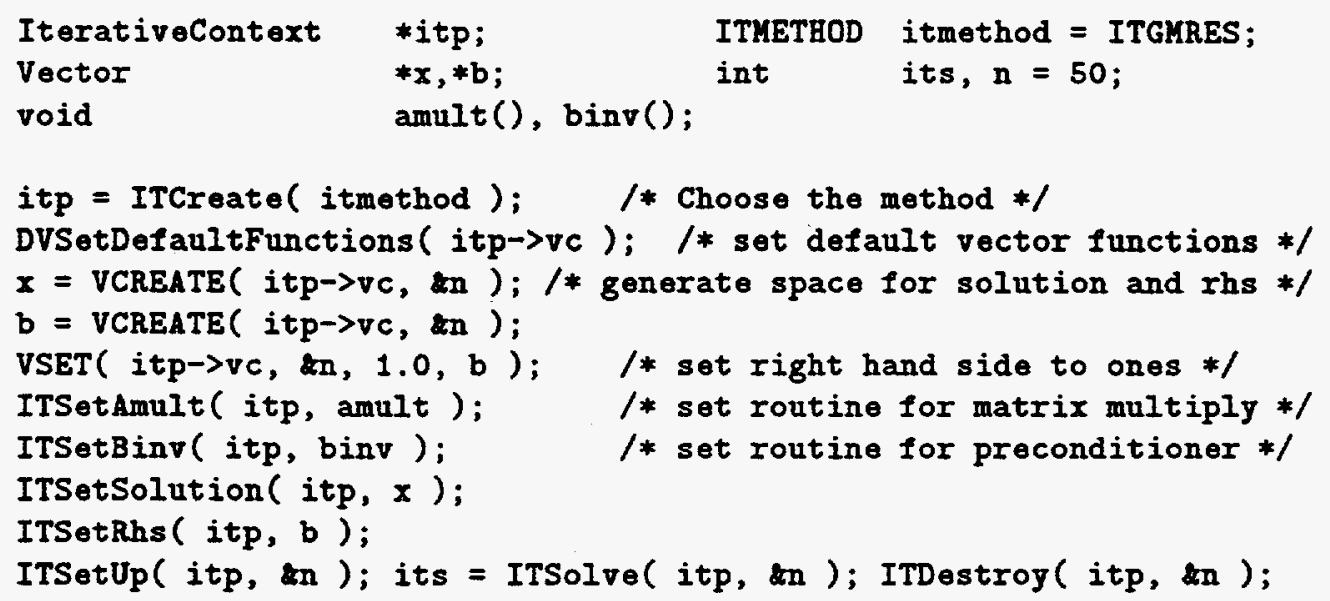

Figure 4: Sample Code Using Krylov Solvers

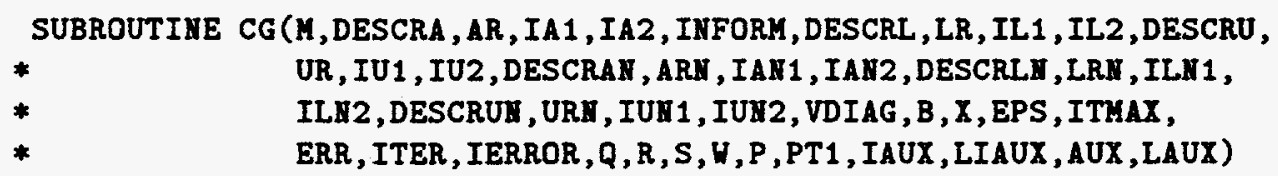

Figure 5: Calling Sequence for a Conjugate Gradient Routine in Fortran 77

were then able to use all of our iterative routines without change. The same approach was used for the parallel version of this application.

Another example is in the EAGLE code [4] for external two- and three-dimensional fluid dynamics. In this code, a linear system must be solved within the inner loop. However, the matrix is represented implicitly as coefficients on a grid. The conventional approach to interfacing this code to a solver package is to reformat the matrix into some explicit representation, such as the YSMP format. With our package, we simply added a new sparse matrix type, "Eagle," that is defined by the grid coefficients and a few operations. This simplified the task of using our package in an existing application. Perhaps more important, it minimized the amount of additional memory needed, since we did not have to make a separate copy of the matrix elements.

Both of these applications codes are written in Fortran 77, demonstrating that the advantages of true object-oriented design can be made available to Fortran users.

We make the following recommendations for the design of truly data-structureneutral libraries:

1. Do not design the interface based on the limitations of the target language. Just because you cannot implement an interface in Fortran does not mean that you cannot provide that interface to Fortran programmers.

2. Do not assume any particular format in the data structures. Do not assume that vectors are contiguous in computer memory (this is not true even in many serial applications codes). 
3. Design the interface so that routines that solve the same problem in different ways are perfectly interchangeable. This approach maximizes the upward compatibility of solutions (new algorithms).

4. Remember that data-structure-neutral does not mean that the format of the matrix is unspecified; it means specifying vectors and matrices and other objects by the operations that are performed on them in such a way that you can operate on them without knowing their internal structure.

Developing the codes initially takes slightly longer than writing one-use, data-structuredependent codes, but the payoff in code reuse more than compensates. Our codes that use these techniques are available via anonymous ftp from the site info.mcs.anl.gov in the directory pub/pdetools. (We support only "double" as the Scalar, mostly for reasons of limited resources.) These routines are callable from both $\mathrm{C}$ and Fortran 77. The linear solvers are part of a larger set of tools, PETSc (Portable, Extensible Tools for Scientific computing) that we have been developing. We have written a set of linear solvers built on top of these libraries called the "Simplified Linear Equation Solvers," SLES. Its user guide [3] is in the file sles.ps.z. SLES is intended as a easy to use, serial interface to the much more powerful underlying routines. We have found this approach to be very easy to use, and we use it both with our research codes and with large computational science applications.

\section{References}

[1] I. S. DUfF, M. MARRONE, AND G. RADICATl, A proposal for user level sparse $B L A S$, Tech. Rep. TR/PA/92/85, CERFACS, 1992. SPARKER Working note \# 1.

[2] S. C. Eisenstat, H. C. Elman, M. H. Schultz, and A. H. Sherman, The (new) Yale Sparse Matrix Package, Tech. Rep. YALE/DCS/RR-265, Department of Computer Science, Yale University, Apr. 1983.

[3] W. D. Gropp AND B. SMITh, Simplified linear equation solvers users manual, Tech. Rep. ANL-93/8, Mathematics and Computer Science Division, Argonne National Laboratory, Mar. 1993.

[4] J. S. Mounts, D. M. BelK, AND D. L. Whitfield, Program EAGLE user's manual, volume IV: Multiblock implicit, steady-state Euler code, Tech. Rep. TR88-117, Vol. IV, Air Force Armanent Laboratory (AFATL), Eglin Air Force Base, Florida, Sept. 1988.

[5] Y. SAAD, SPARSKIT: A basic toolkit for sparse matrix computations, Tech. Rep. 1029, Center for Supercomputing Research and Development, University of Illinois at Urbana-Champaign, Aug. 1990.

The submitted manuscript has been authored by a contractor of the U.S. Government under contract No. W-31-109-ENG-38. Accordingly, the U.S. Government retains a nonexclusive, royalty-free license to publish or reproduce the published form of this contribution, or to allow others to do so, for U.S. Government purposes. 